\title{
MARKERS OF THE EPITHELIAL-MESENCHYMAL TRANSITION IN CELLS OF ENDOMETRIAL CARCINOMA
}

\author{
I.P. Nesina, N.P. Iurchenko, L.G. Buchynska \\ R.E. Kavetsky Institute of Experimental Pathology, Oncology and Radiobiology, \\ NAS of Ukraine, Kyiv 03022, Ukraine
}

\begin{abstract}
Aim: To study the expression of adhesion markers (E-cadherin, $\beta$-catenin and vimentin) associated with epithelial-mesenchymal transition (EMT) and their role in progression of endometrial carcinoma (EC). Materials and Methods: Expression of E-cadherin, $\beta$-catenin and vimentin was studied immunohistochemically in the samples of surgical material of 55 EC patients stage I-III. The proliferation index was determined by flow cytometry. Results: In the group of vimentin-negative EC, tumors of low differentiation grade and deep invasion in myometrium as well as high expression of E-cadherin and $\beta$-catenin prevailed compared with the cases with high expression of vimentin. In addition, in EC with high expression of vimentin, an increase in the number of cells with expression of E-cadherin in the cytoplasm $(78.9 \pm 3.6 \%)$ and $\beta$-catenin with cytoplasmic-nuclear localization $(73.7 \pm 3.2 \%)$ was observed compared with these indices in vimentin-negative tumors $(45.4 \pm 4.2 \%, p<0.001$ and $54.5 \pm 2.6 \%$, respectively, $p<0.005)$, which may indicate EMT-associated changes in EC with high expression of vimentin. Conclusions: The progression of the endometrioid carcinoma may occur in the setting of various molecular changes, in particular, with decreased expression of E-cadherin and $\beta$-catenin and high expression of vimentin, or in the absence of vimentin, utilizing other mechanisms of regulation of proliferative and metastatic potential.
\end{abstract}

Key Words: endometrial cancer, proliferation index, E-cadherin, $\beta$-catenin, vimentin.

According to available literature, endometrial cancer is predominantly diagnosed at early stages $(80 \%$ at stage I) and, as a rule, has a relatively favorable course with a five-year survival of over $95 \%$. However, the five-year survival rates of such patients are much lower in the presence of metastases in regional lymph nodes or relapse of the disease (respectively, $68 \%$ and $17 \%$ ) [1]. In a number of cases, this is due to the low effectiveness of therapeutic measures that do not take into account the molecular mechanisms of cancer progression.

It is known that the biological features of endometrial carcinoma $(E C)$ are formed under the influence of a number of different factors, such as the hormonal background in which tumors have developed, changes in the functioning of oncogenes and suppressor genes, and the composition of the tumor microenvironment. All this contributes to the emergence of genome instability and the accumulation of genetic alterations in cancer cells [2-5].

To some extent, the progression of malignant neoplasms (increased proliferative activity, invasive, angiogenic and metastatic potential) depends on the changes in the expression of molecules that provide intercellular contacts. The loss of adhesive properties by tumor cells contributes to their increased mobility and, as a consequence, penetration into surrounding tissues, blood and lymphatic vessels [6]. Recent studies have shown that the migration of neoplastic cells during tumor progression can be the result of consistent

Submitted: September 04, 2018.

${ }^{\star}$ Correspondence: E-mail: laboncogen@gmail.com Abbreviations used: EC - endometrial carcinoma; EMT - epithelial-mesenchymal transition; IHC - immunohistochemistry; PI proliferation index. molecular changes and a complex of morphological alterations that are accompanied by the destruction of the basement membrane of cancer cells and the loss of the polar orientation of their nuclei, while epithelial cells may acquire mesenchymal-like morphology. This phenomenon was called epithelial-mesenchymal transition (EMT) and, according to Qin et al. [7] is a strategy of immune rescue, adaptation of cancer cells to increase their chances of survival in mesenchymal tissues by mimicry. In this case, abnormal epithelial cells not only lose the expression of epithelial markers, but they may acquire expression of an unusual protein for epithelial cells, a marker of mesenchymal cells - vimentin.

The role of vimentin in determining the malignancy of solid tumors of various genesis has not been clarified yet [8], however, the appearance of this protein in EC cells is associated with tumor invasiveness and metastasis [9]. In contrast, in endometrial serous carcinomas, which are more aggressive tumors with an unfavorable course of the disease, vimentin expression is usually absent or is significantly lower than in endometrioid type EC [10, 11].

The main proteins that provide stable intercellular contacts between epithelial cells and inhibit their mobility are cadherins and catenins, in particular Ecadherin and $\beta$-catenin. E-cadherin belongs to the family of transmembrane glycoproteins being one of the key cadherins that provides $\mathrm{Ca}^{2+}$-dependent homophilic adhesion in epithelial tissues by the formation of transdimers in two adjacent cells via the interaction of extracellular domains [12]. Its intracellular domain is bound to a number of proteins and, in the first place, with $\beta$-catenin, which, apart from E-cadherin, binds with $\alpha$-catenin and actin microfilaments, providing dense intercellular contacts $[12,13]$. The interaction of E-cadherin with $\beta$-catenin is regulated 
by the level of serine and threonine phosphorylation in $\beta$-catenin [14-16].

In most epithelial neoplasias, including EC, there is a decrease in the expression of $\mathrm{E}$-cadherin, including hypermethylation of the promoter of its gene $-\mathrm{CDH} 1$, sometimes with gene mutations or deletions on the $3^{\text {rd }}$ chromosome [17, 18]. In addition, the functional loss of E-cadherin may be due to the activation of transcription factors Snail 1 and Snail2 or ZEB1 and ZEB2, which suppress the expression of the $C D H 1$ gene $[14,19]$. As a result, the destruction of $E$-cadherin/ $\beta$-catenin complex and intercellular bonds occurs, E-cadherin is translocated into the cytoplasm, and $\beta$-catenin is released and accumulated in the cytoplasm followed by its translocation into the nucleus $[13,14]$.

The aim of the study was to analyze the features of the expression of adhesion markers E-cadherin, $\beta$-catenin and vimentin and their role in progression of ECs.

\section{MATERIALS AND METHODS}

The study was conducted on the samples of surgical material of $55 \mathrm{EC}$ patients (47 with stage I and II EC and 8 with stage III EC according to TNM and FIGO classifications). The patients did not receive preoperative therapy, their age ranged from 32 to 73 years (average $58.9 \pm 6.5$ years). All patients were treated at the Department of Oncogynecology of the National Cancer Institute of the Ministry of Health of Ukraine from 2014 to 2017 and provided informed consent on the use of their biological material for scientific research. Morphological diagnosis was verified on histological preparations stained with hematoxylin and eosin. The differentiation grade of tumors was determined according to WHO criteria (2014) [20].

Immunohistochemical (IHC) detection of E-cadherin, $\beta$-catenin and vimentin was performed on deparaffined sections, using MoAbs to E-cadherin (clone $\mathrm{NCH}-38$ ), $\beta$-catenin (clone $\beta$-catenin-1) (DakoCytomation, Denmark), vimentin (clone V9) (Diagnostic BioSystems, the Netherlands). In each slide $700-1000$ cells were analyzed. The results of the $\mathrm{IHC}$ reaction were evaluated by the semiquantitative method counting the number of stained cells and the intensity of the reaction (H-score, points). The analysis took into account the number of cells (\%) with the membrane-cytoplasmic localization of E-cadherin and cytoplasmic-nuclear - of $\beta$-catenin $[9,21]$.

The proliferation index (PI, \%, i.e. the number of cells in the $\mathrm{S}+\mathrm{G} 2 / \mathrm{M}$ phases of the mitotic cycle) was determined using the flow cytometry method after staining cells with fluorochrome - propidium iodide [22]. The studies were conducted on a flow cytometric analyzer EPICS-XL (Beckman Coulter, USA).

To objectivize the evaluation of the expression of the biomolecular markers examined, we determined the median (Me) of the number and intensity of stained cells (H-score) or the proliferative potential $-\mathrm{MePI}$. The values of all indices less than Me were considered low, and above Me - high.

Statistical data were processed using Statistica 8.0 software package (StatSoft, Inc.) by non- parametric Mann - Whitney $U$ Test. Differences at $p<0.05$ were considered significant.

\section{RESULTS}

According to morphological analysis of histological preparations, all patients had endometrioid form of EC of high $(7.2 \%)$, moderate $(41.8 \%)$ and low $(50.9 \%)$ differentiation grade. $40 \%$ of tumors had minor invasion in myometrium $(<1 / 2)$ and $60 \%$ invaded by $>1 / 2$ of myometrium.

According to the results of flow cytometry, it was found that while $\mathrm{S}+\mathrm{G} 2 / \mathrm{M}$ ratio varied from $8.5 \%$ to $48.7 \%$, PI was $27.6 \pm 4.3 \%$, with $\mathrm{Me}=25.4 \%$.

The results of IHC study showed that positive expression of E-cadherin was observed in $78.2 \%$ of $E C$, $\beta$-catenin $-100 \%$ and vimentin in $74.5 \%$. The protein product was detected diffusely, or in separate sections of the tumor (Fig. 1).

At that, a significant variation in the expression of the investigated proteins was observed: individual variations in the expression of $\mathrm{E}$-cadherin were $14.6-190.6$ points (66.3 \pm 5.3 points on the average), $\beta$-catenine $55.0-237.4$ points ( $155.0 \pm 6.4$ points on the average), vimentin $-15.4-242.1$ points ( $53.4 \pm 4.8$ points on the average). Me values of the expression of these markers were $51.5,152.5$ and 41.6 points, respectively.

Comparison of expression of EMT-associated molecular markers in EC of different grade showed that the expression of E-cadherin was higher, and vimentin lower (significantly or at the level of tendency) in lowdifferentiated tumors than in tumors deeply invaded the myometrium (Table 1).

Table 1. Expression of E-cadherin, $\beta$-catenin and vimentin in $\mathrm{EC}$ related to clinical and pathological characteristics of tumors

\begin{tabular}{|c|c|c|c|}
\hline \multirow{2}{*}{ Parameter } & \multicolumn{3}{|c|}{ Expression of markers of EM transition, $\mathrm{H}$-score, points } \\
\hline & E-cadherin & $\beta$-catenin & Vimentin \\
\hline$M \pm m$ & $66.3 \pm 5.3$ & $155.0 \pm 6.4$ & $53.4 \pm 4.8$ \\
\hline Min-Max & $14.6-190.6$ & $55.0-237.4$ & $15.4-242.1$ \\
\hline \multicolumn{4}{|l|}{$\mathrm{Pl}:$} \\
\hline$<\operatorname{Me}(25.4 \%)$ & $52.9 \pm 4.2$ & $159.9 \pm 6.1$ & $52.7 \pm 3.8$ \\
\hline$>\operatorname{Me}(25.4 \%)$ & $93.9 \pm 4.7^{\star \star}$ & $148.3 \pm 6.2$ & $57.1 \pm 4.9$ \\
\hline \multicolumn{4}{|c|}{ Differentiation grade: } \\
\hline G1 & $49.7 \pm 5.4$ & $190.0 \pm 9.3$ & $99.1 \pm 8.4$ \\
\hline $\mathrm{G} 2$ & $77.1 \pm 6.0^{*}$ & $150.0 \pm 8.5^{\star}$ & $56.3 \pm 4.6^{\star}$ \\
\hline G3 & $75.6 \pm 6.1^{\star}$ & $151.9 \pm 8.9^{*}$ & $39.1 \pm 3.3^{\star \star}$ \\
\hline \multicolumn{4}{|c|}{$\begin{array}{l}\text { Depth of tumor inva- } \\
\text { sion into myometrium: }\end{array}$} \\
\hline$<1 / 2$ & $69.1 \pm 4.0$ & $161.0 \pm 9.3$ & $68.4 \pm 4.1$ \\
\hline$>1 / 2$ & $83.3 \pm 5.0$ & $150.7 \pm 9.7$ & $42.5 \pm 3.2^{\star \star}$ \\
\hline \multicolumn{4}{|l|}{ Stage: } \\
\hline I & $73.6 \pm 4.1$ & $149.6 \pm 7.7$ & $66.0 \pm 3.4$ \\
\hline$\|$ & $72.3 \pm 4.6$ & $158.4 \pm 10.2$ & $40.5 \pm 5.7$ \\
\hline III & $92.0 \pm 5.7$ & $171.3 \pm 9.6$ & $14.4 \pm 4.4^{\star \star}$ \\
\hline
\end{tabular}

Notes: E-cadherin: ${ }^{\star} p<0.05$ compared with G1 tumors; ${ }^{\star \star} p<0.01$ compared with $\mathrm{PI}<\mathrm{Me}$; $\beta$-catenin: ${ }^{\star} p<0.05$ compared to $\mathrm{G} 1$ tumors; vimentin: ${ }^{\star} p<0.05$ compared with G1 tumors ${ }^{* \star} p<0.01$ compared to G1 tumors, with invasions in myometrium $<1 / 2$, with stage $\mathrm{I}$.

Expression of the studied markers was significantly reduced in stage III EC compared with these at stages I and II of the disease.

The direction of changes in $\beta$-catenin expression in the investigated tumors of the endometrium was different, namely, lower than Me in tumors of moderate and low differentiation grades and neoplasms with deep 

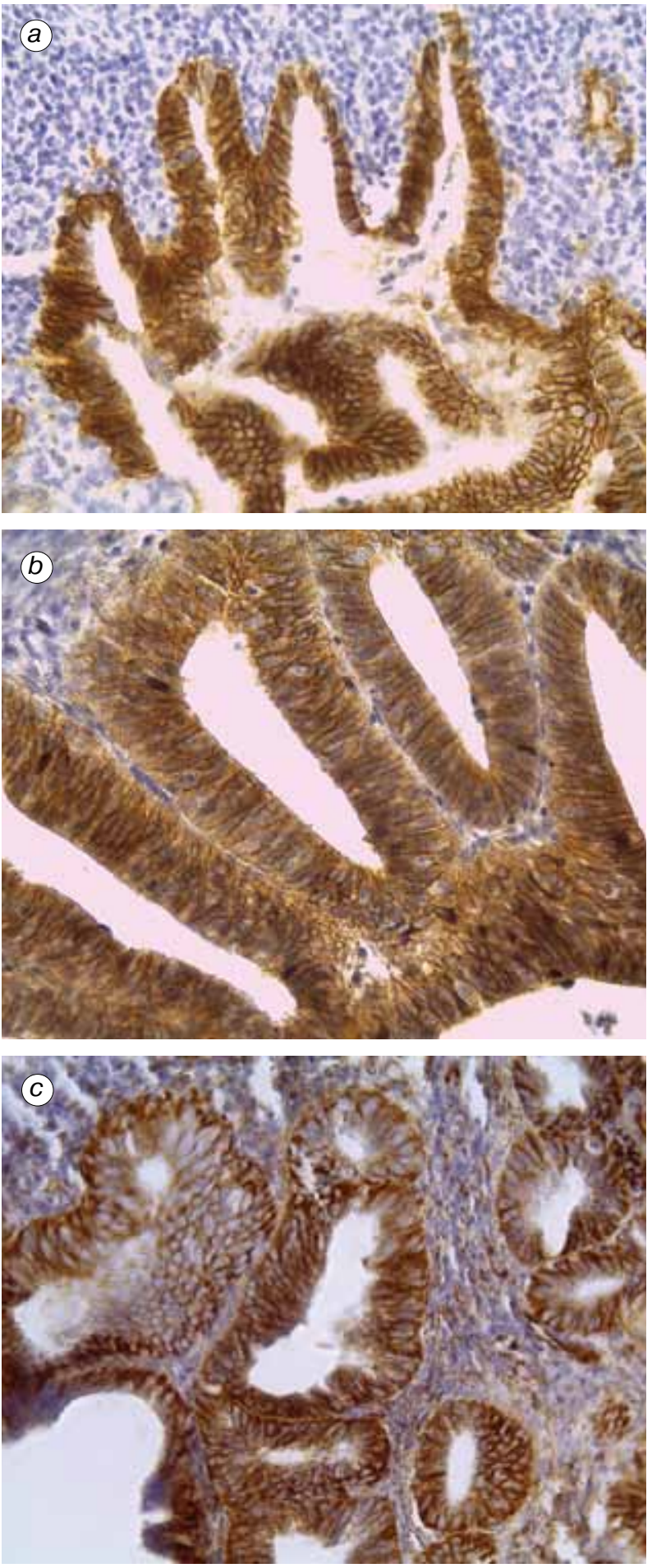

Fig. 1. Expression of E-cadherin and vimentin in the moderately differentiated and $\beta$-catenin in highly differentiated EC, $\times$ 400: $a$ - diffuse membrane-cytoplasmic expression of E-cadherin; $b$-diffuse membrane-cytoplasmic expression of $\beta$-catenin; $c-$ expression of vimentin. IHC, stained with Mayer's hematoxylin invasion into myometrium, and in patients with stage I of the tumor process.

In high-proliferating tumors, the expression of $E$-cadherin was significantly increased, and $\beta$-catenin tended to decrease compared to those in tumors with low PI. Instead, the expression of vimentin practically did not depend on PI index.

Taking into account the available literature, according to which the detection of vimentin in epithelial tumors is one of the characteristics of EMT and the absence or low expression of this protein is more common in serous carcinoma of the endometrium [9, 10], we have compared clinical morphological and molecular-biological indices in tumors of patients with high expression of vimentin and with vimentin-negative EC (Table 2).

The analysis of the data showed that in the group of vimentin-negative $\mathrm{EC}$, tumors of low differentiation grade and deep invasion in myometrium prevailed compared with the cases with high expression of vimentin. In addition, in the tumors with high expression of vimentin, a significant decrease in the expression of E-cadherin was observed as well as the number of cells expressing $\mathrm{E}$ cadherin in the cytoplasm $(78.9 \pm 3.6 \%)$ and $\beta$-catenin with cytoplasmic-nuclear localization (73.7 $\pm 3.2 \%)$ compared to these indices in vimentin-negative tumors (respectively, $45.4 \pm 4.2 \%, p<0.001$ and $54.5 \pm 2.6 \%, p$ $<0.005)$. It was found that in EC without E-cadherin expression, high expression of vimentin (76.9 \pm 6.8 points) was observed, which was higher than in tumors with high expression of E-cadherin (56.1 \pm 4.6 points) .

In tumors without vimentin expression, a high correlation was found between the expression of E-cadherin and $\beta$-catenin $(r=0.7, p<0.05)$, which was absent in the vimentin-positive tumors suggesting the shift in $\mathrm{E}$-cadherin/ $\beta$-catenin ratio.

It should be noted that the proliferative potential in vimentin-negative tumors and tumors with high expression of vimentin was higher than Me. That is, most EC are highly proliferating tumors.

\section{DISCUSSION}

The study revealed a significant EC heterogeneity by clinical and morphological characteristics, and such molecular characteristics as the expression of markers providing intercellular adhesion and associated with EMT. It is known that tumor progression is associated with the acquisition of invasive and metastatic properties by tumor cells. The latter arise as a result of protein-protein and protein-DNA interactions in a number of signaling cascades, which leads to a decrease in intercellular adhesion, which determines the EMT and accelerates the growth rate of the tumor [19].

Table 2. Comparative study of clinical-morphological and molecular-biological indices in EC related to the level of vimentin expression

\begin{tabular}{|c|c|c|c|c|c|c|c|c|}
\hline \multirow{3}{*}{$\begin{array}{c}\text { Expression } \\
\text { of vimentin, } \\
\text { points }\end{array}$} & \multicolumn{5}{|c|}{ Number of cases, $\%$} & \multicolumn{3}{|c|}{ Expression of markers, $\mathrm{M} \pm \mathrm{m}$, points } \\
\hline & \multicolumn{3}{|c|}{ Differentiation grade } & \multicolumn{2}{|c|}{$\begin{array}{c}\text { Depth of tumor invasion into } \\
\text { myometrium }\end{array}$} & \multirow[t]{2}{*}{ E-cadherin } & \multirow[t]{2}{*}{$\beta$-catenin } & \multirow[t]{2}{*}{$\mathrm{Pl}, \%$} \\
\hline & G1 & $\mathrm{G} 2$ & G3 & $<1 / 2$ & $>1 / 2$ & & & \\
\hline \multicolumn{9}{|l|}{$>\mathrm{Me}(41.6)$} \\
\hline $\begin{array}{c}(\mathrm{n}=25) \\
0\end{array}$ & 12.0 & 64.0 & 24.0 & 48.0 & 52.0 & $63.6 \pm 9.4^{*}$ & $151.0 \pm 9.4$ & $27.6 \pm 2.7$ \\
\hline$(n=14)$ & 0 & 35.7 & 64.3 & 21.4 & 78.6 & $98.8 \pm 7.9$ & $160.5 \pm 9.5$ & $28.9 \pm 3.7$ \\
\hline
\end{tabular}

Note: ${ }^{\star} p<0.05$ compared with the vimentin-negative tumors. 
According to current data, one of the causes of EMT may be a hormonal imbalance, in particular a decrease in the progesterone content. For example, van der Horst et al. [23] showed that progesterone stimulates the activity of intratumoral T-lymphocytes and inhibits the Wnt/ $\beta$-catenin pathway associated with EMT. It is possible that, as was shown in our previous studies, the low level of progesterone in the peripheral blood of EC patients, along with the low expression of progesterone receptors in tumors and the increase in the number of intratumoral FOXR3 lymphocytes in low-differentiated EC, created conditions for EMT, which led to a deep invasion of myometrium [24-26]. In fact, in some EC samples under study, EMT-associated changes (low or no expression of E-cadherin, translocation of E-cadherin into the cytoplasm, and $\beta$-catenin - into the nucleus) may be supposed, which is consistent with the literature data [13-15, 27, 28].

These translocations of E-cadherin and $\beta$-catenin can occur during activation of various signaling cascades (TGF $\beta$, FGF and Wnt), and due to disorders in the functioning of the genes of $C D H 1, C T N N D 1$ or genes that provide degradation of $\beta$-catenin (e.g., APC gene) $[13,14]$. In a nucleus, a transcriptional complex formed between $\beta$-catenin and T-cell- and lymphoid factors (TCF LEF) activates the expression of a number of target genes, including the C-MYC, CCND1, VEGF, Axin-2, Snail, VIM and others, which leads to an increase in proliferative and angiogenic potential of malignant neoplasms, which is accompanied by the appearance of a marker of the mesenchymal tissues vimentin $[13,14,16,19]$.

Increased expression of vimentin associated with an increase in the migration and invasive properties of cancer cells was noted by other researchers in malignant neoplasms and cell lines derived from the tumors of other genesis $[11,29]$. The presence of such a link has been shown in cell lines and surgical samples of breast tumors with low estrogen receptor levels. The authors emphasize that the expression of vimentin influences the invasive phenotype of cancer cells only in cooperation with other, yet clearly unidentified proteins [30].

Separate attention should be given to the cases of EC with high-level expression of E-cadherin and $\beta$-catenin, and without expression of vimentin. In $64.3 \%$ of cases, such tumors were characterized by a low differentiation grade and in $78.6 \%$ of cases - by a deep invasion in myometrium. That is, more than $60 \%$ of these tumors were more aggressive than vimentin-positive tumors. It is possible that in vimentin-negative tumors, could be present a EGFR-mediated activation of PI3K/ AKT signaling, which is accompanied by an increase in the expression of E-cadherin and the formation of tumor cell complexes - microembolies with dense intercellular contacts with so-called "collective or chain migration" $[12,14]$.

Summing up the results obtained, it can be hypothesized that the formation of a more aggressive phenotype in the vimentin-negative tumors of the endometrium is not due to the reduction of the expression of the markers of intercellular adhesion $\mathrm{E}$-cadherin and $\beta$-catenin, but via the changes in the functioning of a number

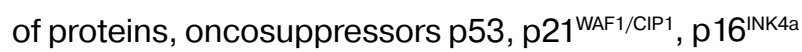
and others [11, 31, 32].

To confirm our experimental data, a bioinformatics analysis of the VIM mRNA expression was performed [33]. The portal Oncomine, which contains data on microarrays and RNA sequencing, was used. The evaluation of the array data showed that VIM mRNA expression in EC of various differentiation grades and the stages at the level of mRNA is similar to our results obtained during $\mathrm{IHC}$ analysis of vimentin expression.

The level of VIM mRNA expression in moderately and low-differentiated EC is reduced in comparison with highly differentiated tumors (Fig. 2).

In addition, it has been shown that VIM expression also progressively decreased in tumors of patients with stage II and III compared to that in patients with stage I (Fig. 3).

Thus, the data of the bioinformatics analysis show that the decrease in the expression of vimentin in EC correlates with the aggressiveness of this type of cancer.

In conclusion, our data show that the progression of the endometrioid carcinoma may occur due

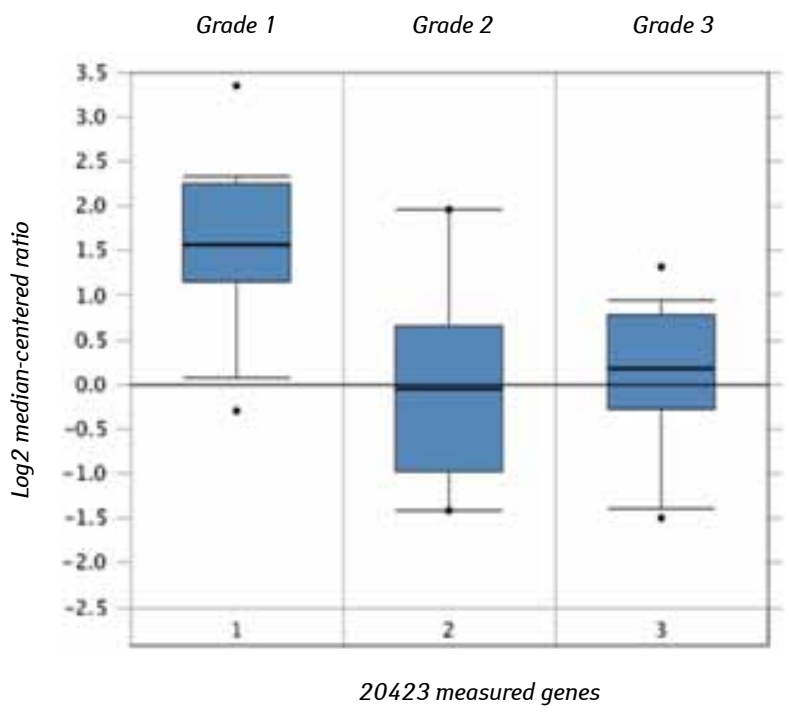

Fig. 2. Expression of the VIM gene at the level of mRNA in EC of varying differentiation grades (Grade 1-3)

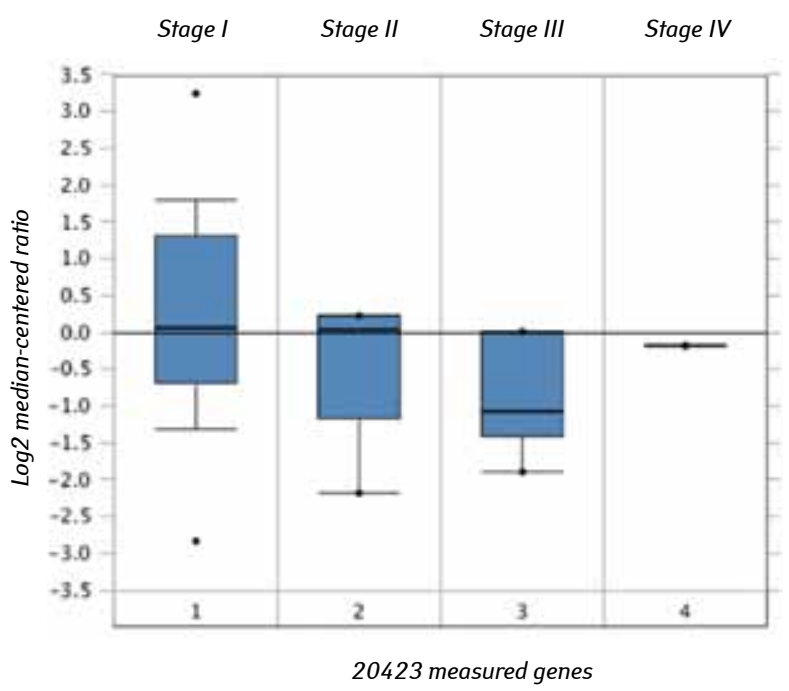

Fig. 3. Expression of the VIM gene at the level of mRNA in EC carcinomas stages I-IV by FIGO 
to various molecular changes, in particular, decreased expression of $\mathrm{E}$-cadherin and $\beta$-catenin proteins, and high expression of the marker of mesenchymal tissues - vimentin, or in the absence of vimentin expression, with the involvement of other mechanisms of regulating proliferative and metastatic potential.

\section{ACKNOWLEDGMENTS}

Authors are grateful to S.V. Nespryadko, MD (National Cancer Institute of $\mathrm{MH}$ of Ukraine) for providing tissue samples of EC.

\section{REFERENCES}

1. Colombo N, Creutzberg C, Amant F, et al. ESMO-ESGO-ESTRO Consensus Conference on endometrial cancer: diagnosis, treatment and follow-up. Ann Oncol 2016; 27: 16-41.

2. Ashrafian LA, Kiselev VI. Tumors of the reproductive organs (etiology and pathogenesis). Moscow: Dimitrade Gafic Group, 2007. 214 p. (in Russian).

3. Hanley KZ, Birdsong GG, Mosunjac MB. The recent developments in surgical pathology of corpus uteri. 2017. Arch Pathol Lab Med 2017: 141: 528-41.

4. Leeman EE, Mukhina MS. Cellular microenvironment of malignant tumors and its significance in the prognosis. Vopr Onkol 2013; 59: 444-52 (in Russian).

5. Nesina IP, Iurchenko NP, Nepryadko SV, Buchynska LG. Tumor-associated macrophages, FOXP3 ${ }^{+}$-lymphocytes and dense microvessels in endometrial carcinoma of patients with varying states of menstrual function. Onkologiya 2017; 19: 52-6 (in Ukrainian).

6. Ribatti D. Epithelial-mesenchymal transition in morphogenesis, cancer progression and angiogenesis. Exp Cell Res 2017; 353: 1-5.

7. Qin J-H, Wang L, Li Q-L, et al. Epithelial-mesenchymal transition as strategic microenvironment mimicry for cancer cell survival and immune escape? Genes Diseases 2017; 4: 16-8.

8. Qin Z, Kreplak L, Buehler MJ. Hierarchical structure controls nanomechanical properties of vimentin intermediate filaments. PLoS ONE 2009; 4: e7294.

9. Desouki MM, Kallas SJ, Khabele D, et al. Differential vimentin expression in ovarian and uterine corpus endometrioid adenocarcinomas: diagnostic utility in distinguishing double primaries from metastatic tumors. J Gynecol Pathol 2014; 33: 274-81.

10. Irving AJ, Hsu F, Dupuis B, et al. Immunoprofile of cervical and endometrial adenocarcinomas using a tissue microarray. Virchows Arch 2003; 442: 271-7.

11. Hemalatha A, Suresh TN, Kumar ML. Expression of vimentin in breast carcinoma, its correlation with Ki67 and other histopathological parameters. Indian J Cancer 2013; 50: 189-94.

12. Glushankova NA, Zhitnyak IYu, Aiollo DV, Rubtsova $\mathrm{SN}$. The role of E-cadherin in the neoplastic evolution of epithelial cells. Usp Mol Oncol 2014; 1: 12-7 (in Russian).

13. Isaeva AV, Zima AP, Shabalova IP, et al. $\beta$-Catenin: structure, function and role in cancer transformation of epithelial cells. Vestnik RAMS 2015; 70: 47583 (in Russian).

14. Gall TMH, Frampton AE. Gene of the month: Ecadherin (CDH1). J Clin Pathol 2013; 66: 928-32.

15. Minkhovich MV, Midiber KYu, Gallyamova AR, et al. Immunohistochemical evaluation of cadherin-catenin complex expression in breast cancer. Zh Anat Histol 2017; 6: 63-8 (in Russian).
16. Valenta T, Hausmann G, Basler K. The many faces and functions of $\beta$-catenin. EMBO J 2012; 31: 12714-36.

17. Markowska A, Pawalowska M, Lubin I, Markowska J. Signalling pathways in endometrial cancer. Contemp Oncol 2014; 18: 143-8.

18. Park JH, Lee BI, Song ES, et al. Hypermethylation of E-cadherin in endometrial carcinoma. J Gynecol Oncol 2008; 19: 241-5.

19. Makker A, Goel MM. Tumor progression, metastasis, and modulators of epithelial-mesenchymal transition endometrioid endometrial carcinoma: an update. Endocr Relat Cancer 2016; 23: 85-111.

20. Kurman RJ, Carcangiu ML, Herrington CS, Young RH. WHO Classification of Tumours of the Female Reproductive Organs (IARC WHO Classification of tumours). 4th Ed: IARC 2014. 307 p.

21. Korolenkova LI, Stepanova EV, Ermilova VD, et al. Expression of E-cadherin is a biochemical marker of the progression of the disease in cervical intraepithelial neoplasms. Vestn Mosk University, Ser. 2. Chemistry 2012; 2: 272-7 (in Russian).

22. Nicoletti G, Migliorati M, Pagliacci C, et al. A rapid and simple method for measuring thymocyte apoptosis by propidium iodide staining and flou cytometry. J Immunol Meth 1991; 139: 271-9.

23. van der Horst PH, Wang Y, Vandenput I, et al. Progesterone inhibits epithelial-to-mesenchymal transition in endometrial cancer. Plos ONE 2012: 7: e30840.

24. Nesina IP, Iurchenko NP, Brieieva OV, et al. The level of steroid hormones in the serum of patients with endometrial cancer with different states of menstrual function and indices of the genome instability. Onkologiya 2016; 18: 210-5 (in Ukrainian).

25. Buchynska LG, Iurchenko NP, Nesina IP, et al. The status of steroid hormones receptors in endometrial carcinomas as an important prognostic indicator in patients at postmenopausal period. Onkologiya 2016; 68: 123-9 (in Ukrainian).

26. Iurchenko NP, Glushchenko NM, Buchynska LG. Comprehensive analysis of intratumoral lymphocytes and FOXP3 expression in tumor cells of endometrial cancer. Exp Oncol 2014; 36: 353-8.

27. Clarke BA, Gilks CB. Endometrial carcinoma: controversies in histopathological assessment of grade and tumour cell type. J Clin Pathol 2012; 63: 410-5.

28. Florescu MM, Pirici D, Simionescu CE, et al. E-cadherin and $\beta$-catenin immunoexpression in endometrioid endometrial carcinoma. Rom J Morphol Embryol 2016; 57: 1235-40.

29. Wei J, Xu G, Wu M, et al. Overexpression of vimentin contributes to prostate cancer invasion and metastasis via src regulation. Anticancer Res 2008; 28: 327-34.

30. Satelli A, Li S. Vimentin in cancer and its potential as a molecular target for cancer therapy. Cell Mol Life Sci 2011; 68: 3033-46.

31. Buchynska LG. Endometrial cancer: taxonomy of genetic changes of tumor cells and their role in determination of malignant potential. Extended Abstract of Doct Sci Dissertation Kyiv, 2012. 34 p.

32. Yusuda M. Immunohistochemical characterization of endometrial carcinomas: endometrioid, serous and clear cell adenocarcinomas in association with genetic analysis. J Obstet Cynaecol Res 2014; 40: 2167-76.

33. Compendia bioscience (http://www.oncomine.com). TCGA (The Cancer Genome Atlas). Endometrial adenocarcinoma gene expression data. Platform not pre-defined in Oncomine Measured 20,423 genes, 111,123 reporters Office 